\title{
Intake of 3 Eggs/Day or Equivalent Amount of Choline as Supplement for 4 Weeks Increases Plasma Choline Without Changing Plasma TMAO or Microbiota diversity in Participants with Metabolic Syndrome
}

\author{
Minu Sarah Thomas, Marissa Dibella, Christopher N Blesso and Maria-Luz Fernandez * \\ Objective: The purpose of this study was to compare equivalent amounts of two choline sources on \\ plasma choline and its derivatives including trimethylamine $\mathrm{N}$-oxide (TMAO), inflammatory \\ markers and changes in fecal microbiota in men and women aged 32 to 70 years old with metabolic \\ syndrome (MetS).
}

Methods: Twenty-three subjects with MetS were included in this randomized, crossover clinical trial. Participants underwent an initial period of 2 weeks without consuming any eggs, which was followed by a random allocation to either 3 eggs/day or a choline-supplement for 4 weeks (both diets had a choline equivalent of $400 \mathrm{mg}$ per day). Following a 3-week washout period, participants were allocated to the alternate diet. We measured plasma choline and plasma TMAO as well as Creactive protein (CRP), inflammatory markers, insulin, liver enzymes and analyzed fecal microbiota.

Results: Although there was an overall significant increase in plasma choline after egg intake, compared to baseline $(\mathrm{P}<0.01)$, there were no significant differences between egg and supplement at the end of the respective interventions $(P>0.05)$. Baseline values were $7.9 \pm 2.1 \mathrm{nmol} / \mathrm{ml}$ compared to $9.9 \pm 2.2$ and $9.5 \pm 2.1 \mathrm{nmol} / \mathrm{ml}$ for the egg and supplement, respectively $(\mathrm{P}<0.01)$. In addition, plasma TMAO was not different between baseline, or at the end of the egg and supplement periods $(\mathrm{P}>0.1)$. In regards to inflammatory and insulin resistance markers, $\mathrm{C}$-reactive protein, plasma insulin, and insulin resistance (HOMA-IR) were each lower after the egg period compared to baseline ,"while no differences in these parameters were observed after the supplement period when compared to baseline. In contrast, plasma IL- 6 concentrations were lower by both dietary treatments compared to baseline. There were no significant differences in fecal bacterial diversity when baseline was compared to either the egg or the choline supplement treatment by using the Shannon diversity index or when time and treatment were compared. Some interesting observations were that the family of Lachnosipiracae (Phylum Bacteroidetes) appeared to increase after choline treatment (both after the supplement and the egg period). Also, Akkermansia (Phylum Verrucomicrobia) was higher after both treatments, yet more so after the choline supplement period.

Conclusions. These studies indicate that plasma choline response appears to be similar in MetS participants, independent of its source or chemical composition. Analyzing the microbiota of these subjects also showed no significant differences between treatments indicating that both bioavailability of choline, TMAO production and fecal microbiota composition are not affected by choline source in MetS patients.

Keywords:eggs; choline; metabolic syndrome; TMAO; microbiota 